\title{
Excess nitrogen as a marker of intense dinitrogen fixation in the Western Tropical South Pacific Ocean: impact on the thermocline waters of the South Pacific
}

Alain Fumenia ${ }^{1}$, Thierry Moutin ${ }^{1}$, Sophie Bonnet ${ }^{2}$, Mar Benavides ${ }^{1,4}$, Anne Petrenko ${ }^{1}$, Sandra Helias $5 \quad$ Nunige $^{1}$, and Christophe Maes ${ }^{3}$

\section{Supplementary}

Table S1: Oceanographic cruises available in GLODAPv2 database for the South Pacific. The cruises presented figure 12 are marked with a star.

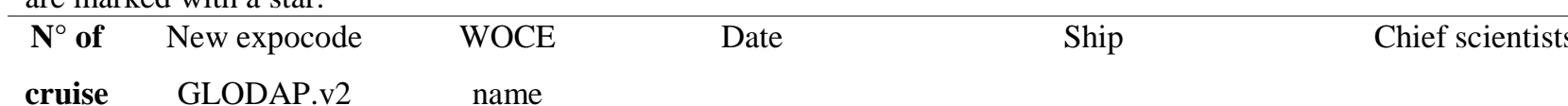

\begin{tabular}{clllll}
\hline $\mathbf{1}$ & 09AR19930404 & P11A & Mar-Apr 1993 & RV Aurora Australis & Rintoul \\
\hline $\mathbf{2}$ & 09FA19930624* & P11S & Jun-July 1993 & RV Franklin & Church \\
\hline $\mathbf{3}$ & 49NZ20072211* & P14C & Oct-Dec 2007 & RV Mirai & Takeshi Kawano \\
\hline $\mathbf{4}$ & 31DS19960105 & P14S P15S & Jan-Mar 1996 & RV Discoverer & Bullister, Johnson, \\
& & & & & Feeley, Roberts \\
\hline
\end{tabular}

\begin{tabular}{|c|c|c|c|c|c|}
\hline 5 & 09FA20010524* & P15 & May-July 2001 & RV Franklin & Wijffels \\
\hline 6 & 31WT19910831* & P16C & Aug-Oct 1991 & RV T. Washington & Talley \\
\hline 7 & 325020060213 & P16N_2006 & Feb-Mar 2006 & RV Thomas.G. Thompson & Sabine \\
\hline 8 & 31WT19910716* & P16S & July Aug 1991 & RV T. Washington & Swift \\
\hline 9 & $316 N 19920901 *$ & P16A-P17A & Oct-Nov 1992 & RV Knorr & Reid \\
\hline 10 & 33RR20050109 & P16S_2005 & Jan-Feb 2005 & RV Roger Revelle & Sloyan \\
\hline 11 & 31DS19940126* & P18 & Jan-Apr 1994 & RV Discoverer & Taft, Johnson \\
\hline 12 & 33RO20071215 & P18_2007 & Dec-Jan 2007 & RV Ronald H. Brown & Bullister, Johnson \\
\hline 13 & $316 \mathrm{~N} 19930222 *$ & P19 & Feb-Apr 1993 & RV Knorr & Talley \\
\hline 14 & 316 N19920502 & P06 & May-July 1992 & RV Knorr & $\begin{array}{l}\text { Bryden, McCartney, } \\
\text { Toole, Whoi }\end{array}$ \\
\hline 15 & 49NZ20030803 & P06_2003 & Aug-Oct 2003 & RV Mirai & Watanabe, Fukasawa \\
\hline 16 & 318M20091121* & P06_2009 & Nov 09- Feb 2010 & RV Melville & Curry, Macdonald, \\
\hline 17 & 318M19940327 & P21_1994 & Mar-May 1994 & RV Melville & Bryden, McCartney \\
\hline 18 & 49NZ20090410* & P21_2009 & Apr-June 2009 & RV Mirai & Murata, Uchida \\
\hline 19 & 318M19730822 & I & Aug 73-June 1974 & RV Melville & $\begin{array}{l}\text { Weiss, Brewer, Biscaye, } \\
\text { Takahashi, Broecker, } \\
\text { Edmond, Craig }\end{array}$ \\
\hline 20 & $325019940125^{*}$ & I & Jan-Feb 1994 & RV Thomas.G. Thompson & Dean, Roemmich \\
\hline 21 & 31OC19870606* & I & June-July 1987 & RV Oceanographer & Stanley, Hayes \\
\hline 22 & 49HН20011127* & I & Nov 01-Jan 2002 & RV Hakuho-Maru & Terazaki \\
\hline 23 & 325020131025 & GT13 & Oct-Dec 2013 & RV Thomas.G. Thompson & German, Cutter \\
\hline
\end{tabular}




\section{Figures caption}

Fig. 1. Map of depths (dbar) on isopycnal surface 24.7 (a), and of isopycnal surface 26.3 (b). The data come from GLODAPv2 database (Table S1). Continuous black lines represent major large-scale currents in the South Pacific (details are available in figure 2).

Fig. S2. Mean $\mathrm{N}^{*}\left(\mu \mathrm{mol} \mathrm{kg}{ }^{-1}\right)( \pm \mathrm{sd})$ between longitude $\left[150^{\circ} \mathrm{E}-180^{\circ}\right],\left[180^{\circ}-150^{\circ} \mathrm{W}\right],\left[150^{\circ} \mathrm{W}-120^{\circ} \mathrm{W}\right],\left[120^{\circ} \mathrm{W}-90^{\circ} \mathrm{W}\right]$, $\left[90^{\circ} \mathrm{W}-80^{\circ} \mathrm{W}\right]$ and between latitude $\left.\left.\left.\left[5^{\circ} \mathrm{S}-15^{\circ} \mathrm{S}\right], 15^{\circ} \mathrm{S}-23^{\circ} \mathrm{S}\right], 23^{\circ} \mathrm{S}-32^{\circ} \mathrm{S}\right], 32^{\circ} \mathrm{S}-45^{\circ} \mathrm{S}\right]$.

Fig. S3. $\mathrm{N}^{*}\left(\mu \mathrm{mol} \mathrm{\textrm {kg } ^ { - 1 } )} v s\right.$. density $\left(\mathrm{kg} \mathrm{m}^{-3}\right)$ for different sections of GLODAPv2 database.

Fig. S4. $\mathrm{N}^{*}\left(\mu \mathrm{mol} \mathrm{kg}{ }^{-1}\right)$ on $\sigma 24.7$ (green line) and on $\sigma 26.3$ (black line) vs latitude for (a) sections (P14C 1991), (b) section P14S P15S (1996), (c) section P16S (2005) and (d) P18 (2007). Detail of sections is available in table S1.

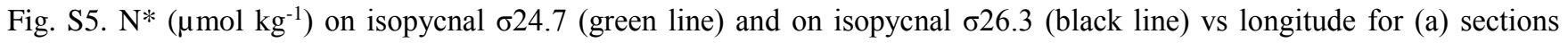
35 (cruise 20, 1994), (b) section GT13 (2013), (c and d) section P21 (1994) and (2009), (e and f) section P06 (1992) and (2003). Detail of sections is available in table $\mathrm{S} 1$. 


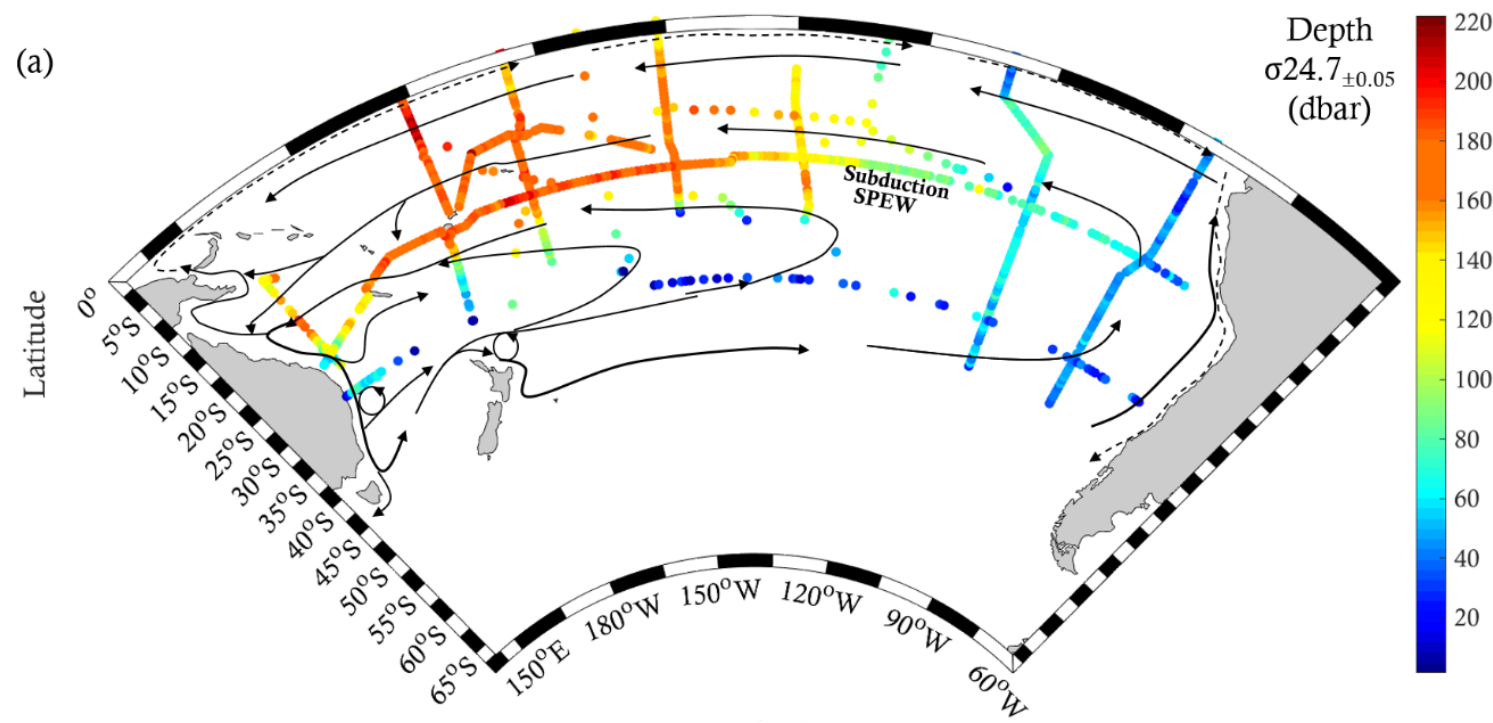

(b)

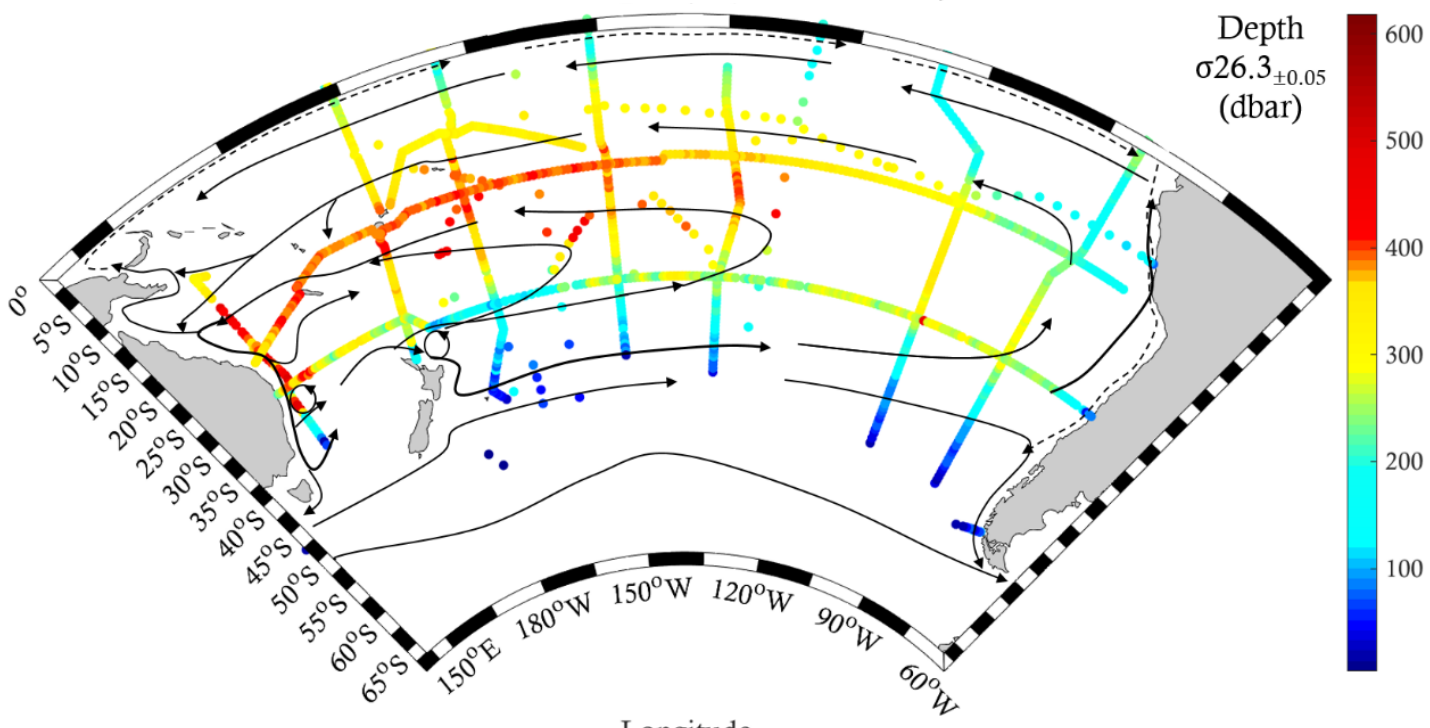

Figure S1. a.b. 


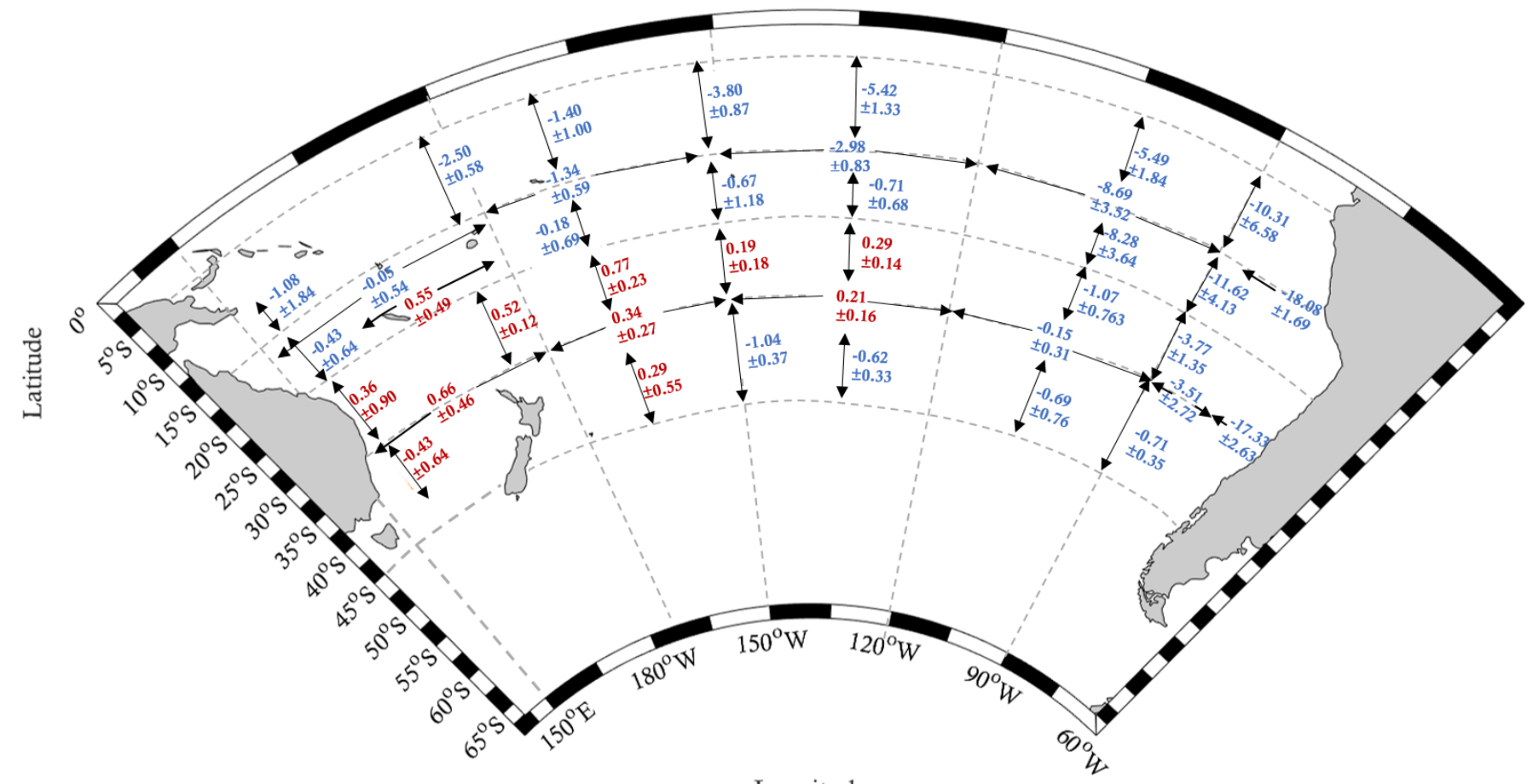

Longitude

Figure S2 

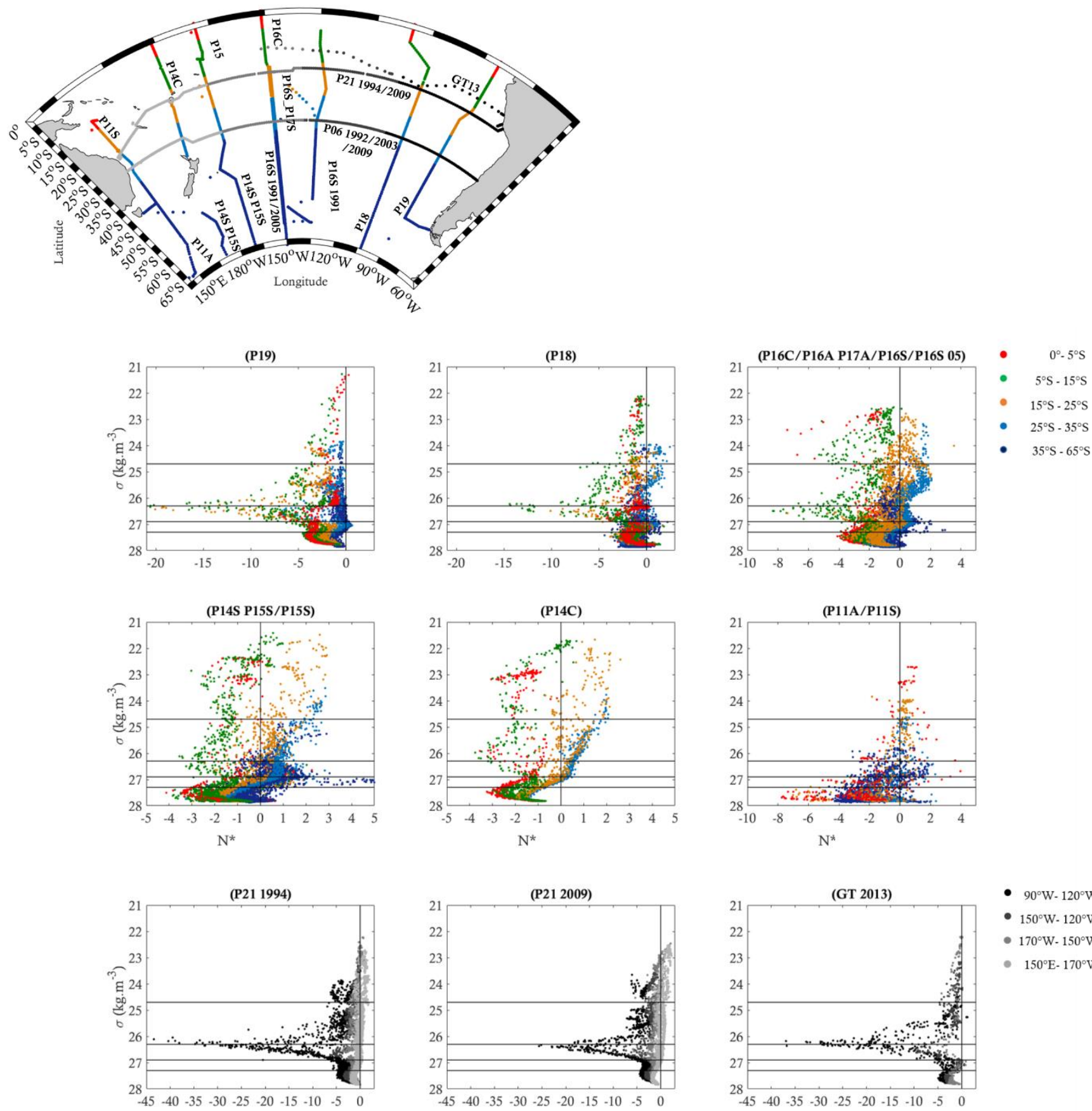

- $90^{\circ} \mathrm{W} \cdot 120^{\circ} \mathrm{W}$

- $150^{\circ} \mathrm{W}-120^{\circ} \mathrm{W}$

- $170^{\circ} \mathrm{W}-150^{\circ} \mathrm{W}$

- $150^{\circ} \mathrm{E}-170^{\circ} \mathrm{W}$
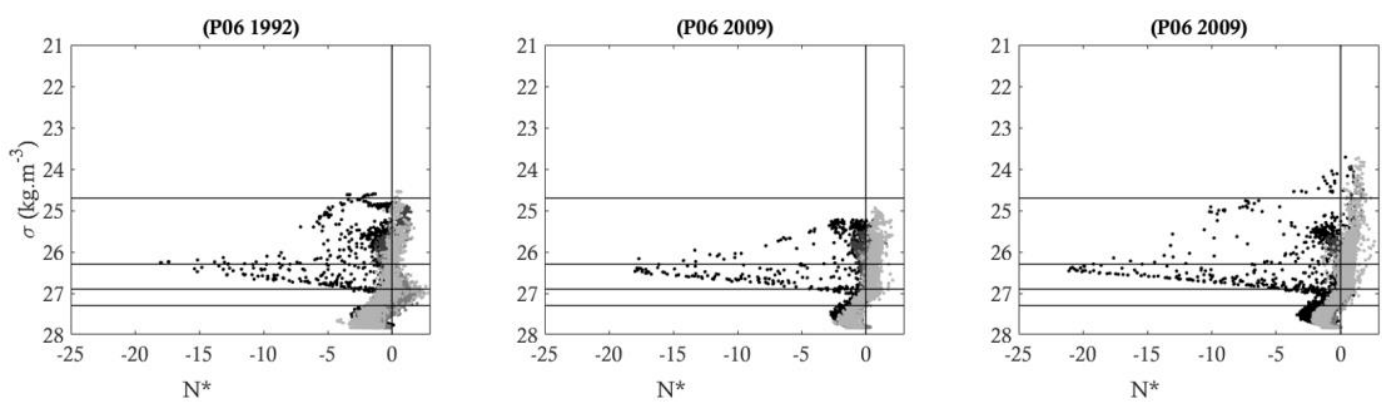

Figure S3 

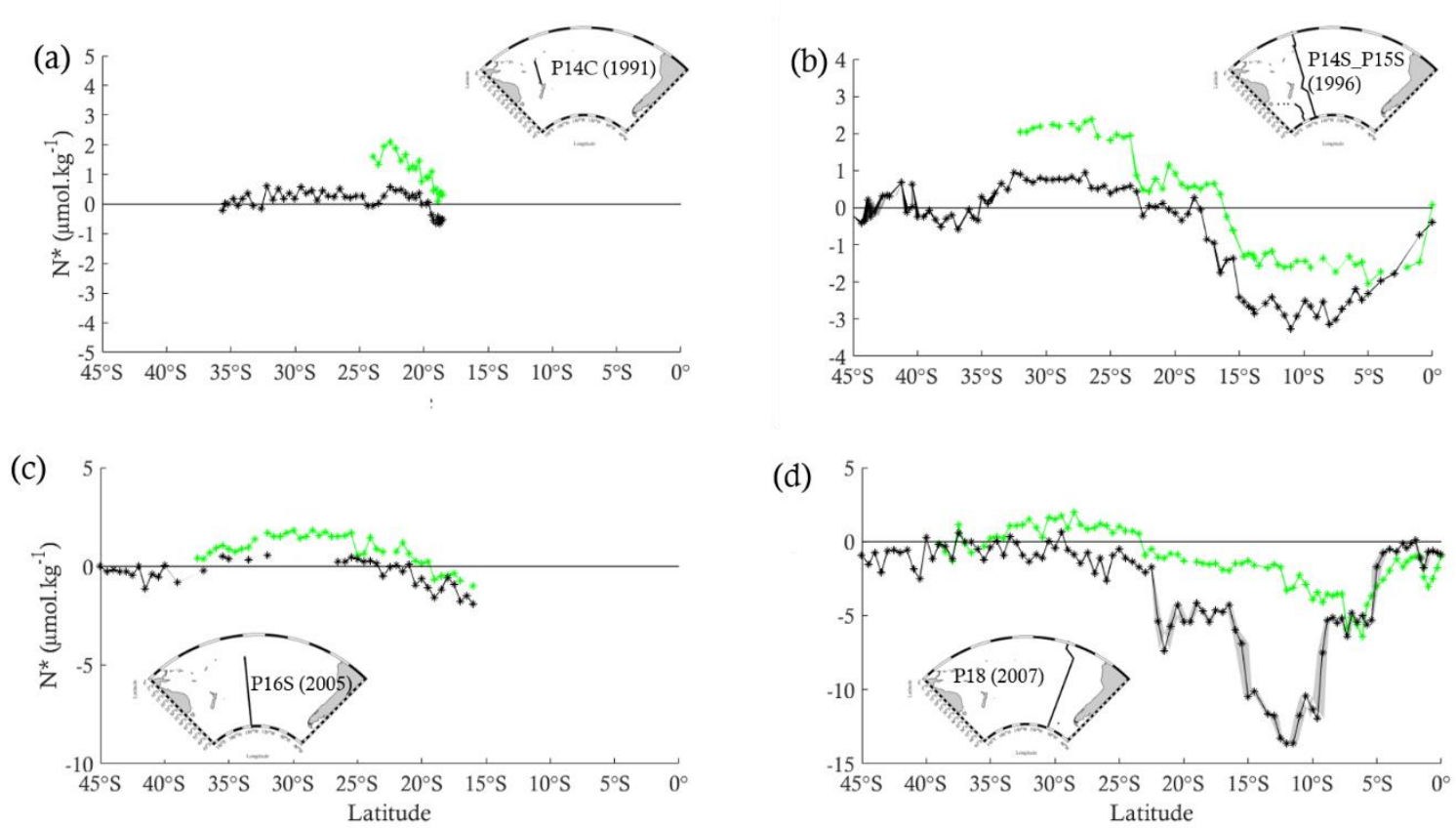

Figure S4. a.b.c.d. 


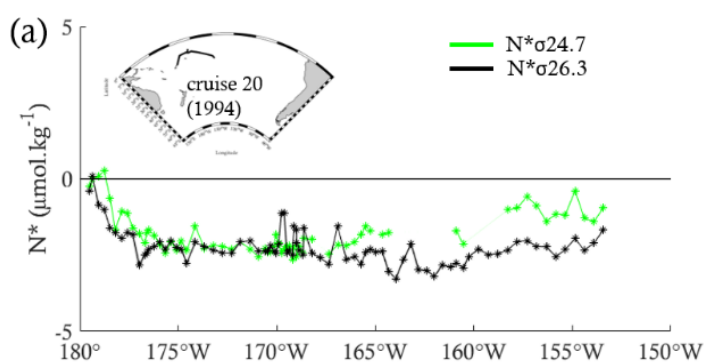

(b)
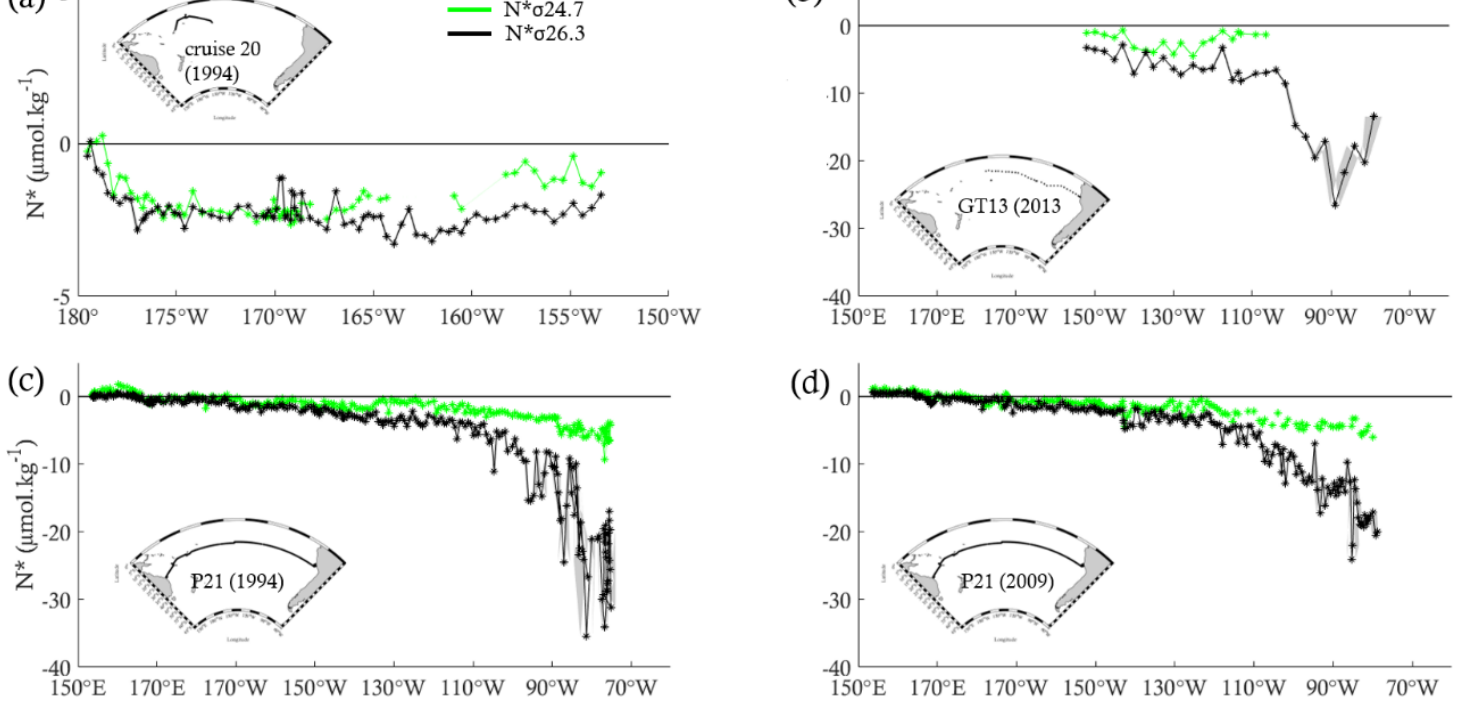

(d)
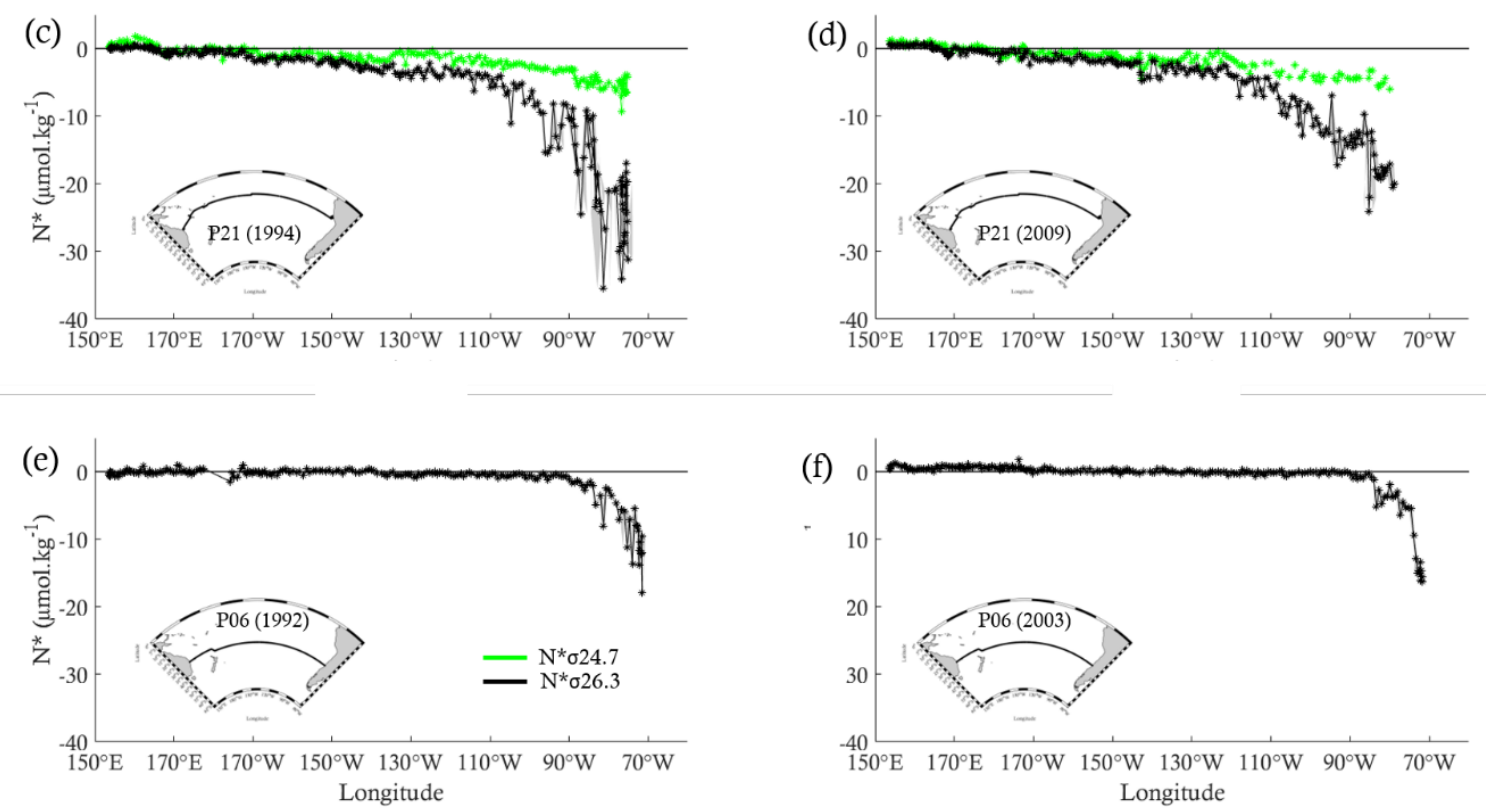

Figure S5. a.b.c.d.e.f 